\title{
Vierfachtherapie wirkt besser gegen H. pylori
}

\author{
In der Behandlung von H.-pylori-Infektionen gilt seit diesem Jahr ein neuer Standard: \\ Demnach soll sich die Eradikationstherapie künftig auch an Risikofaktoren für eine \\ Clarithromycinresistenz orientieren.
}

\begin{abstract}
Die bisherigen Empfehlungen zur Behandlung von H.-pylori-Infektionen sahen bei entsprechender Indikation die Dreifachkombination aus Protonenpumpenhemmer (PPI), Clarithromycin und entweder Amoxicillin oder Metronidazol als Standardtherapie der ersten Linie vor. Dieses als „französische“ bzw. „italienische" Tripeltherapie bekannte Schema führt nach neueren Untersuchungen jedoch nur bei etwa $73 \%$ der Patienten zum Erfolg. Häufige Ursache für ein Therapieversagen sind die zunehmenden Resistenzen gegenüber Clarithromycin.

Eine große Metaanalyse hat ergeben, dass sich mit einer Vierfachtherapie in verschiedenen Kombinationen (u. a. PPI + 3 Antibiotika; PPI + Bismut + 2 Antibiotika) in 85-94\% der Fälle eine Eradikation des Magenkeims erzielen lässt [BMJ 2015;351:h4052]. Die Quadrupeltherapie ist denn auch Bestandteil der neuen S2k-Leitlinie „Helicobacter pylori und gastroduodenale Ulkuskrankheit“.
\end{abstract}

\section{Bei V.a. Clarithromycinresistenz zusätzlich Bismut}

Nach Prof. Wolfgang Fischbach, Aschaffenburg, gilt es in Zukunft vor einer Eradikationstherapie nach Risikofaktoren für eine Clarithromycinresistenz zu fahnden. Patienten, die aus Regionen mit hohen Resistenzraten stammen (z. B. Südoder Osteuropa) oder anamnestisch eine Makrolidtherapie erhalten haben, gelten nach Fischbach als Risikokandidaten für eine Resistenz. Sie sollte man über zehn Tage mit der Bismut-basierten Vierfachtherapie (PPI + Bismut + 2 Antibiotika) behandeln. Alternativ kommt hier auch eine bismutfreie Vierfachtherapie über sieben Tage infrage (PPI + alle drei Antibiotika der Dreifachtherapie).

„Liegen solche Risikofaktoren nicht vor, können Sie unverändert die klassi-

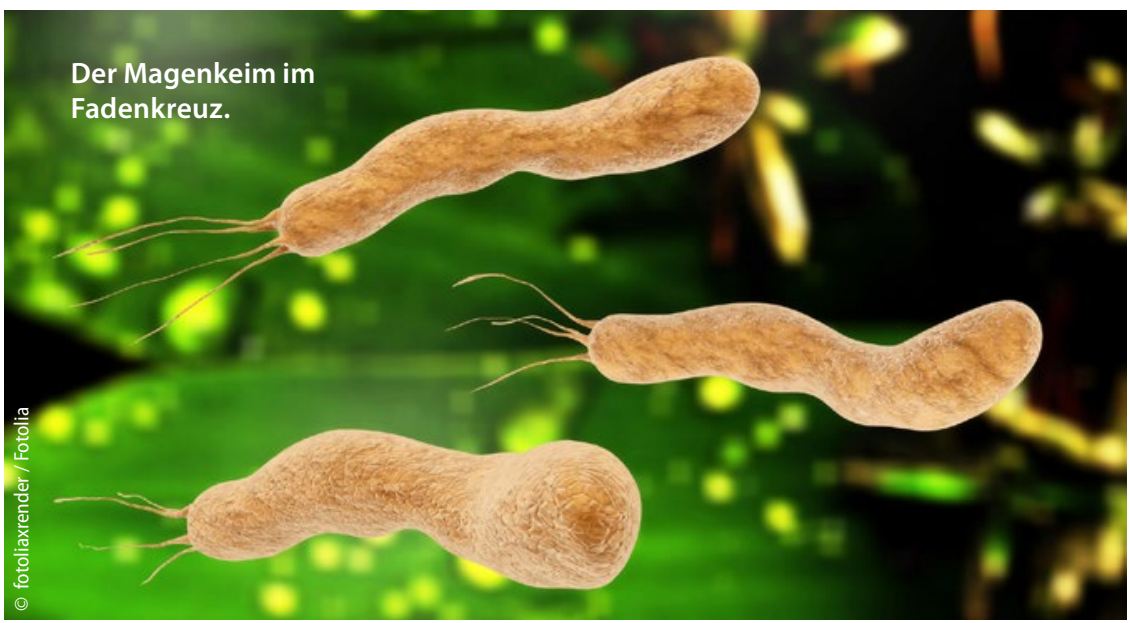

sche französische oder italienische Tripeltherapie anwenden“. Dabei sei eine Behandlung über 14 Tage der einwöchigen Therapie vorzuziehen. Unabhängig vom Resistenzrisiko könne man sich jedoch auch für die Bismut-basierte Quadrupeltherapie entscheiden. Für den Fall eines Therapieversagens empfiehlt die Leitlinie eine Fluorchinolon-haltige Tripeltherapie.

\section{Wann ist die Eradikation indiziert?}

Als Indikationen zur Keimeradikation gilt laut Leitlinie der Nachweis eines peptischen Ulcus ventriculi oder duodeni, das gastrale MALT-Lymphom, die idiopathische thrombozytämische Purpura, der Morbus Ménétrier und die lymphozytäre Gastritis - jeweils bei Nachweis einer H.-pylori-Infektion!

Für die funktionelle Dyspepsie besteht bei entsprechendem Keimnachweis eine Kann-Empfehlung. Refluxsymptome stellen dagegen per se keine Indikation für eine H.-pylori-Eradikation dar.

\section{Risikofaktor NSAR}

Wie Fischbach betonte, erhöht eine Infektion mit $\mathrm{H}$. pylori für sich genommen das Risiko einer Ulkusblutung im Magen um den Faktor zwei. Das Risiko steige auf das Achtfache, wenn zusätzlich NSAR eingenommen werden. Daher empfiehlt die Leitlinie, vor Einleitung einer Langzeittherapie mit NSAR oder auch ASS bei Patienten mit einer Ulkusanamnese einen Test auf H. pylori durchzuführen und bei positivem Ergebnis zu eradizieren (s. nebenstehendes Interview).

\section{PPI schützen}

Auch wenn unter NSAR- oder ASS-Einnahme eine Blutung im oberen Gastrointestinaltrakt eintritt, soll auf H. pylori getestet werden. Fischbach empfahl, in solchen Fällen immer auch einen PPI zu verschreiben. „ASS und NSAR sind anerkannte Risikofaktoren für gastroduodenale Ulzera und ihre Komplikationen“, warnte er. Das Blutungsrisiko werde auch durch Einnahme von Antikoagulanzien erhöht; das gelte sowohl für das alte Phenprocoumon als auch für die neuen oralen Antikoagulanzien (NOAK).

\section{Dr. Elke Oberhofer}

- Praxis Update Allgemeinmedizin, April 2016 in München 